\title{
MEDIÇÃO CONTÍNUA DE TEMPERATURA DO AÇO LÍQUIDO NO DISTRIBUIDOR UTILIZANDO PIRÔMETRO INFRAVERMELHO DE FIBRA ÓPTICA
}

\section{Resumo}

O presente trabalho, desenvolvido na Usiminas, descreve a metodologia, os benefícios, as comparações com outros tipos de medições e os resultados operacionais do sistema de medição contínua de temperatura do aço líquido no distribuidor da máquina de lingotamento contínuo $n^{\circ} 4$ da Aciaria $n^{\circ}$ I. A medição de temperatura utiliza um manipulador mecânico, uma cerâmica de alumina como corpo negro, um pirômetro de fibra óptica e um dispositivo de refrigeração e proteção do sensor. Foram utilizados métodos comparativos através de medição com termopar do tipo " $\mathrm{S"}$ e a experiência operacional para a implementação da nova medição. O sistema de medição contínua de temperatura levou à redução dos custos operacionais, da exposição térmica do operador de plataforma, dos riscos de acidentes pessoais e operacionais e mostra a tendência da variação da temperatura do processo ao longo do tempo.

Palavras-chave: Medição contínua; Lingotamento contínuo; Pirômetro.

\section{CONTINUOUS MEASUREMENT OF LIQUID STEEL TEMPERATURE IN THE TUNDISH BY USING OPTIC FIBER INFRARED PYROMETER}

\begin{abstract}
This paper describes the methodology developed by Usiminas and its benefits of using continuous measurement system of liquid steel temperature in the tundish, at Usiminas continuous casting plant \# 4 of steelmaking \# I, and also makes comparisons with other types of measurements showing its operational results. The temperature measurement system uses an aluminum ceramic as a black body, an optic fiber pyrometer protected by a cooling device, and a mechanism that places the sensor on/off line. Comparative methods using " $S$ " type termocouple and the operational experience were used for the new measurement system implementation. The continuous measurement system has reduced the operator's thermal exposition, risks of accidents and also operational costs. This system shows the temperature variation tendency during casting process.
\end{abstract}

Key words: Continuous measurement; Continuous casting; Pyrometer.

\section{INTRODUÇÃO}

As indústrias siderúrgicas têm conseguido notável progresso nas últimas décadas, na melhoria da qualidade dos seus produtos, aumento da produtividade, melhoria no controle ambiental, redução dos custos operacionais e aumento da segurança pessoal e operacional. Isso tem incentivado de maneira significativa a busca por processos inovadores de produção, desenvolvimento de equipamentos no controle dos processos e medição de seus parâmetros.

No processo de solidificação do aço no lingotamento contínuo, são utilizados basicamente dois tipos de sensores para a medição de temperatura do metal líquido no distribuidor. A maioria das siderúrgicas utiliza termopares de platina do tipo " $\mathrm{S}$ "(I) (10\% PtRh e Pt), montados em pontas descartáveis, para medições eventuais. Uma minoria, devido ao alto custo, utiliza no processo de monitoramento contínuo, termopares de platina do tipo "B" (2) (94\% Pt/6\% Rh e $70 \% \mathrm{Pt} / 30 \% \mathrm{Rh})$, em montagens especiais devidamente protegidos. Esses tipos de medições apresentam índice elevado de manutenção, erros consideráveis na medição, alto custo e riscos de acidentes pessoal e operacional.

Visando minimizar os problemas citados, foi avaliada dentre outros métodos, a aplicação de pirômetro óptico que mostrou-se bastante adequada. ${ }^{(3)}$

Foram utilizados métodos comparativos e a experiência operacional para a implementação e validação da nova medição.

\footnotetext{
'Membro da ABM, Engenheiro de Instrumentação, Gerência de Instrumentação dos Altos Fornos, Aciarias e Energia da Usiminas Ipatinga - MG

${ }^{2}$ Membro da ABM, Gerente, Gerência de Seção de Lingotamento da Aciaria I da Usiminas Ipatinga - MG

${ }^{3}$ Técnico de Instrumentação, Gerência de Instrumentação dos Altos-Fornos, Aciarias e Energia da Usiminas Ipatinga - MG
} 


\section{PRINCÍPIO DE MEDIÇÃO DOS PIRÔMETROS ÓPTICOS}

A radiação infravermelha faz parte do espectro eletromagnético, e inclui vários tipos de ondas como rádio, microondas, luz visível, luz ultravioleta, raios gama e raios $\mathrm{X}$. A faixa infravermelha está entre a porção visível do espectro e as ondas de rádio. Os comprimentos de ondas infravermelhas são geralmente expressos em mícron $(\mu \mathrm{m})$, com o espectro se estendendo de 0,7 micra a I.000 micra. Somente a faixa de 0,7 micra a 18 micra é usada para a medição de temperatura.

A intensidade da energia infravermelha emitida de um objeto aumenta ou diminui em razão da sua temperatura. Emissividade é o termo utilizado para determinar as características de emissão de energia de diferentes materiais e superfícies.

Um pirômetro óptico de duas cores ou de razão, ${ }^{(4)}$ detecta a energia infravermelha de um objeto e focaliza a energia em dois sensores fotossensíveis, incorporados em uma única pastilha de silício. Os detetores convertem a energia infravermelha num sinal elétrico, que é convertido dentro de um valor de temperatura baseado na equação de calibração do medidor e na configuração da inclinação da curva de saída de temperatura (slope). Esse valor de temperatura pode ser exibido no medidor, saindo como sinal elétrico ( $4 \mathrm{~mA}$ a $20 \mathrm{~mA})$ e exibido no computador.

Quando o alvo é muito pequeno ou quando outras partículas interferem no sinal de medição, como fumaça, vapor e poeira, um pirômetro de duas cores ou de razão é mais efetivo.

Os pirômetros ópticos, onde a cabeça está separada dos medidores eletrônicos com um cabo de fibra óptica, são aplicados em locais onde há campos eletromagnéticos ou em outros ambientes desfavoráveis.

A Equação I é a função matemática de saída do pirômetro de fibra óptica de duas cores.

$\mathrm{T}=\left(\mathrm{E}_{\lambda 1} \cdot \lambda_{1} \cdot \varepsilon_{\lambda_{1}} \cdot \mathrm{X}_{1} \%\right) /\left(\mathrm{E}_{\lambda 2} \cdot \lambda_{2} \cdot \varepsilon_{\lambda 2} \cdot \mathrm{X}_{2} \%\right)$

Sendo:

T: temperatura $\left({ }^{\circ} \mathrm{C}\right)$;

$\varepsilon_{\lambda_{1}}=\varepsilon_{\lambda 2}$ : emissividades do tubo cerâmico relativa aos $\lambda_{1}$ e $\lambda_{2}$;

$\lambda_{1}$ : comprimento de onda da medição do sensor I $(\mu \mathrm{m})$;

$\lambda_{2}$ : comprimento de onda da medição do sensor $2(\mu \mathrm{m})$;

$\mathrm{E}_{\lambda \mid}:$ radiação relativa ao $\lambda_{1}$;

$\mathrm{E}_{\lambda 2}$ : radiação relativa ao $\lambda_{2}$; e

$X_{1} \%=X_{2} \%$ : percentual de obstrução (\%).

Em pirômetro de duas cores ou de razão, como é mostrado na equação $I$, o percentual da relação da obstrução $\left(X_{1} \% / X_{2} \%\right)$ é constante e igual a $I$. As emissividades relativas $\left(\varepsilon_{\lambda_{1}}\right.$ e $\left.\varepsilon_{\lambda_{2}}\right)$ são iguais, pois o ponto de leitura é único, então a relação $\varepsilon_{\lambda 1} / \varepsilon_{\lambda 2}$ é também constante e igual a I. Desta forma, a função matemática final de saída do pirômetro de fibra óptica de duas cores, é dada pela Equação 2.

$\mathrm{T}=\mathrm{E}_{\lambda 1} \cdot \lambda_{1} / \mathrm{E}_{\lambda 2} \cdot \lambda_{2}$

O resultado da Equação 2, depende apenas dos valores dos comprimentos de ondas gerados no interior da cerâmica $\left(\lambda_{1}\right.$ e $\left.\lambda_{2}\right)$ e das radiações relativas $\left(E_{\lambda 1}\right.$ e $\left.E_{\lambda 2}\right)$.

\section{DESCRIÇÃO DO SISTEMA DE MEDIÇÃO COM PIRÔMETRO ÓPTICO}

Para a utilização do pirômetro óptico na medição contínua da temperatura do aço líquido no distribuidor, a Usiminas desen- volveu um dispositivo que permitiu o uso dessa tecnologia, originando a patente no Brasil e no exterior de $\mathrm{n}^{\circ} \mathrm{PI}-0502779-9$ de 09 de junho de 2005.

O sistema de medição contínua utiliza um pirômetro de razão com fibra óptica, um conversor óptico/elétrico (4 mA a $20 \mathrm{~mA})$, uma cerâmica de alumina grafitada como corpo negro e uma caixa com refrigeração protegendo a cabeça óptica. A fibra óptica é o meio de transmissão da energia infravermelha emitida no interior da cerâmica, sendo que essa radiação é proporcional à temperatura do processo.

A cerâmica, composta de alumina grafitada e prensada, possui a capacidade de promover uma alta condutividade térmica e luminosa. Para permitir a medição da temperatura do processo, a cerâmica possui um furo longitudinal com uma das extremidades fechada, onde o pirômetro está focado para fazer a leitura. A cabeça óptica está devidamente alinhada dentro da cerâmica de forma a garantir uma leitura precisa, sem as interferências da parede interna da mesma.

O processo de medição consiste basicamente em mergulhar a cerâmica e aquecê-la à temperatura do aço líquido. O pirômetro óptico faz a leitura da radiação instantânea, processa e envia para o sistema de registro e indicação. $O$ tempo inicial necessário para estabilizar a medição é de aproximadamente 6 a 10 minutos.

A vida útil desse tubo cerâmico depende das características do aço em processo. Em média, a cerâmica dura $24 \mathrm{~h}$ de operação, podendo atingir um tempo maior. Na Figura I é apresentado o desenho do dispositivo.

O pirômetro óptico foi montado de forma a não sofrer nenhum dano no momento da troca da cerâmica e da limpeza da lente do sensor, ${ }^{(5)}$ gerando baixo custo operacional e de manutenção.

Através de simulações diretas, realizando-se medições com sensores descartáveis, termopares do tipo "S", foi possível ajustar o tempo de resposta da medição de temperatura do processo, alterando a inclinação da curva de medição (slope) e ajustando o filtro de saída.

Nesse sistema é necessário substituir o tubo cerâmico e fazer a limpeza na lente a cada $24 \mathrm{~h}$ de operação. A atividade de substituição e limpeza da lente é realizada pela operação. A calibração do pirômetro óptico ${ }^{(6)}$ é realizada a cada 12 meses, sendo que até o presente momento não foi verificado nenhum desvio da calibração durante esse intervalo de tempo. 


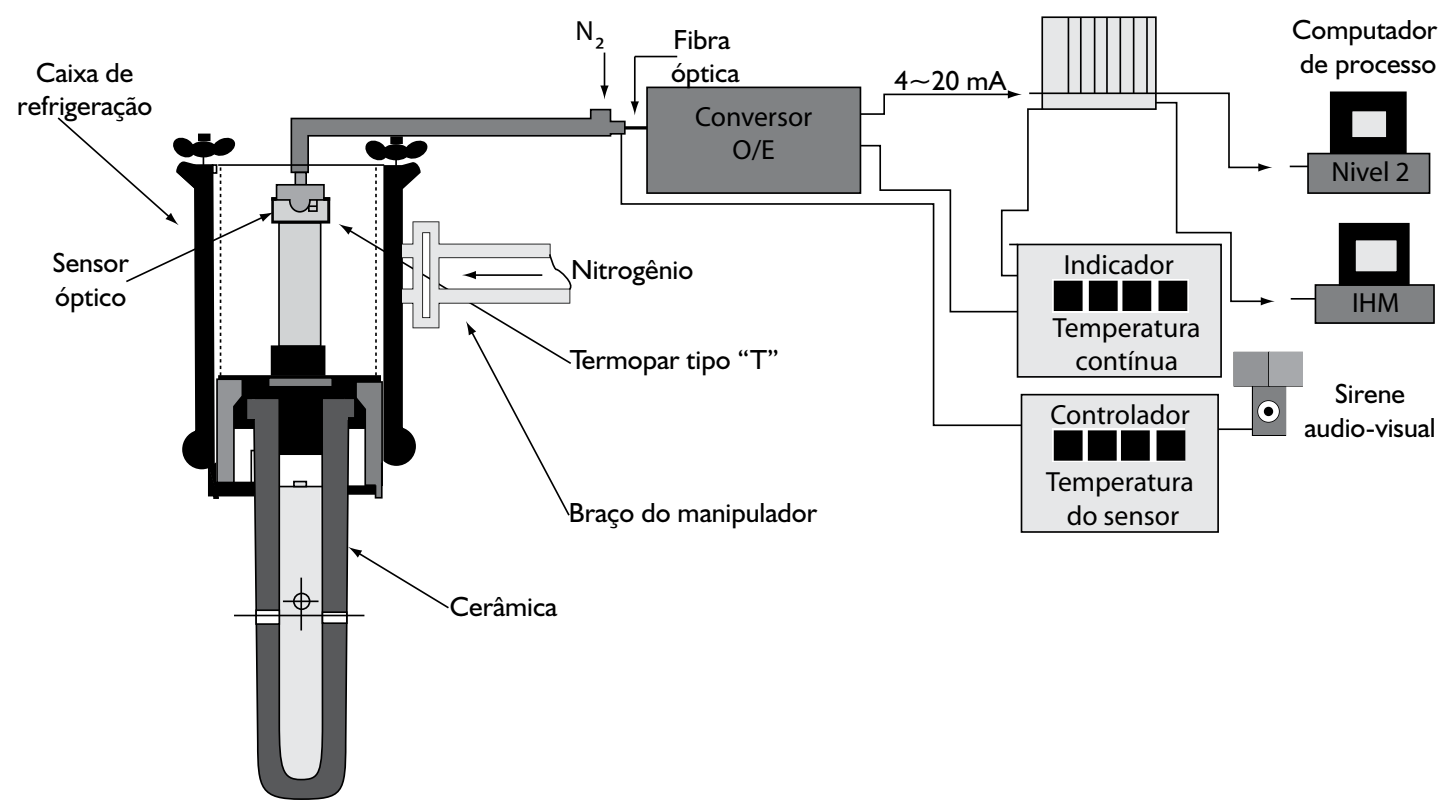

Figura I. Diagrama simplificado do sistema de medição da Usiminas.

\section{VARIÁVEIS IMPORTANTES NO DESENVOLVIMENTO DO PROJETO}

No decorrer desse desenvolvimento, surgiram várias interferências, tais como:

- especificar um pirômetro óptico adequado à aplicação;

- alta temperatura na cabeça óptica do sensor infravermelho, superior a $200^{\circ} \mathrm{C}$;

- monitorar a temperatura na região onde a cabeça óptica está instalada;

- projetar e desenvolver um dispositivo mecânico para instalação e proteção da cabeça óptica;

- tornar a aplicação adequada à operação, garantindo a substituição da cerâmica de forma fácil, rápida e segura;

- estabelecer o diâmetro interno e comprimento do tubo de aço inox para fixar a cabeça óptica;

- manter a fixação e alinhamento da cabeça óptica no tubo de aço inox;

- projetar um novo tubo cerâmico com as dimensões adequadas à necessidade do processo, minimizando os efeitos do alinhamento do conjunto mecânico entre o pirômetro óptico e a cerâmica;

- definir a vazão e pressão do gás de refrigeração para a fibra e a cabeça óptica sem comprometer a medição;

- definir a distância entre cabeça óptica e o fundo da cerâmica;

- minimizar a interferência na medição durante a variação do nível de aço líquido no distribuidor, durante a troca da panela, principalmente em baixa tonelagem; e

- ajustar o tempo de resposta, isto é, a sensibilidade da medição e o fator de correção da inclinação da curva (slope).

Com o objetivo de solucionar essas interferências e viabilizar a aplicação do sensor, foi desenvolvido um sistema utilizando nitrogênio para refrigeração interna da unidade eletrônica, da fibra óptica e da cabeça sensora, minimizando os efeitos da alta temperatura que estão submetidos $\left(>200^{\circ} \mathrm{C}\right)$. A caixa de proteção garante o alinhamento da cabeça óptica e do tubo cerâmico e elimina os efeitos dos impactos operacionais, tais como o manuseio durante a substituição da cerâmica, limpeza da lente e choques mecânicos. Para monitorar a temperatura interna, foi instalado um termopar do tipo "T" $\left(0^{\circ} \mathrm{C}\right.$ a $\left.400^{\circ} \mathrm{C}\right)$ junto à cabeça sensora, ligado a um controlador digital responsável por gerar alarmes sonoros e visuais quando a temperatura ultrapassa um limite previamente estabelecido.

\section{FATORES QUE PODEM INFLUENCIAR NA MEDIÇÃO}

São variáveis que podem influenciar negativamente no resultado da medição:

- sujeira na lente;

- formação de sílica, gerada pelo processo, no interior do tubo cerâmico;

- nível de aço no distribuidor abaixo de 18 toneladas;

- alinhamento da cabeça óptica com o tubo cerâmico;

- fumaça, vapor e poeira em excesso no interior do tubo cerâmico;

- volume de nitrogênio para refrigeração da cabeça sensora; e

- distância entre o sensor óptico e o ponto de medição. 


\section{PRINCIPAIS BENEFíCIOS DA MEDIÇÃO COM PIRÔ- METRO ÓPTICO}

Deve-se destacar que nas malhas de medições que utilizam sensores de platina, como termopares tipo " $\mathrm{S}$ " ou " $\mathrm{B}$ ", a incerteza é superior $\mathrm{a} \pm 8^{\circ} \mathrm{C}$.

A incerteza dos pirômetros ópticos para a faixa de medição de $1.400^{\circ} \mathrm{C}$ a $1.800^{\circ} \mathrm{C}$ é em média $\pm 0,36 \%$ do valor medido. É um dispositivo de medição com baixo índice de manutenção se comparado com termopares.

Na Figura 2 é apresentado o dispositivo de medição após ser retirado do banho.

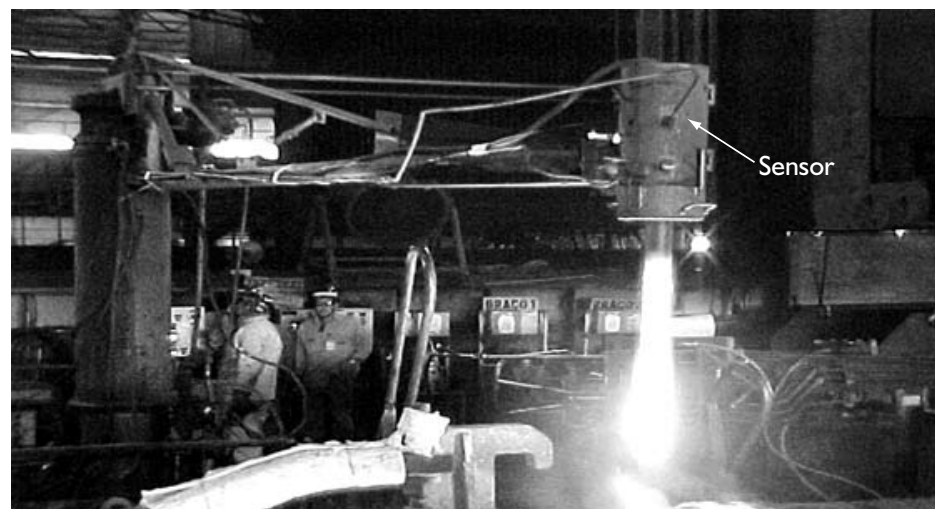

Figura 2. Vista geral do sistema de medição após ser retirado do banho.

Na Figura 3 é apresentado o gráfico dos custos entre os diversos tipos de medição e fabricantes, para dois anos consecutivos de operação, nas quatro máquinas de lingotamento da Usiminas. Os dados incluem a aquisição de equipamentos e instalação, não considerando o desembolso com as atividades de manutenção.

O pirômetro óptico é mais econômico em relação aos outros tipos de medição, sendo que o custo do primeiro ano de utilização é maior em relação ao segundo em função da aquisição de equipamentos e materiais de consumo (cerâmica). $O$ mesmo não ocorre para o segundo ano, pois o desembolso é apenas com a cerâmica.

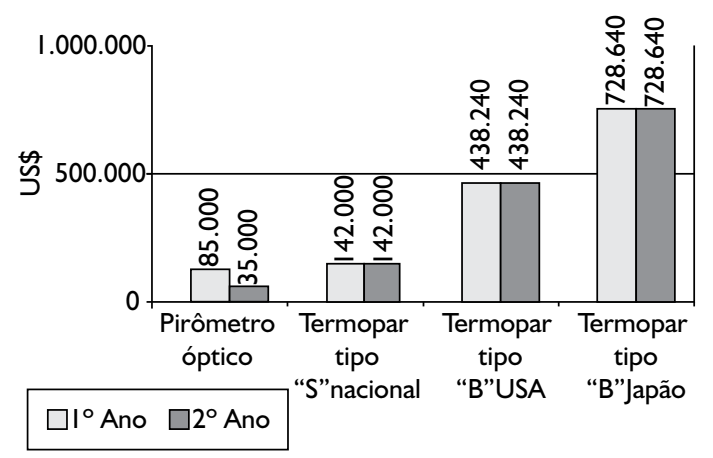

Figura 3. Gráfico de custo entre os diversos tipos de medição.
Os custos para os demais tipos de medição de outros fabricantes utilizados nessa aplicação para o primeiro e segundo anos são iguais, por se tratarem de materiais de consumo constante (termopares, cabos de compensação e cerâmica).

Os dados de custos operacionais e de aquisição de equipamentos apresentados na Figura 3, não levam em consideração as variações dos índices econômicos, tais como inflação e variação cambial.

O dispositivo desenvolvido pela Usiminas possui maior robustez pois foi protegido de tal maneira que fatores externos como altas temperaturas e interferência humana não degradem seu desempenho. A caixa de proteção evita que o sistema fique sujeito a danos durante sua operação e manutenção.

Com a medição contínua é possível um acompanhamento das variações de temperatura do processo, reduzindo problemas operacionais como obstrução das válvulas longa e submersa e rompimento de pele.

Os gases gerados no interior do tubo cerâmico, em função da alta temperatura do processo, maior que $1.500^{\circ} \mathrm{C}$, provocam a oxidação dos termopares de medição contínua. $\mathrm{O}$ mesmo não ocorre com o pirômetro óptico.

Com o novo sistema reduziu-se a necessidade das tomadas sistemáticas de temperatura com lança de imersão manual durante o lingotamento, em média três medições por corrida, consequentemente reduziu-se $o$ tempo de exposição do operador de plataforma à alta temperatura e aos riscos de acidentes, gerando maior conforto pessoal e disponibilidade operacional. A Figura 4 apresenta o operador no momento da medição utilizando a lança de medição manual com sensor descartável, termopar tipo "S".

Nas aplicações da Usiminas, a vida média dos termopares do tipo "B", para a medição contínua, foi de $150 \mathrm{~h}$ de operação. Já a vida útil do pirômetro óptico, até o momento, ultrapassou quatro anos.

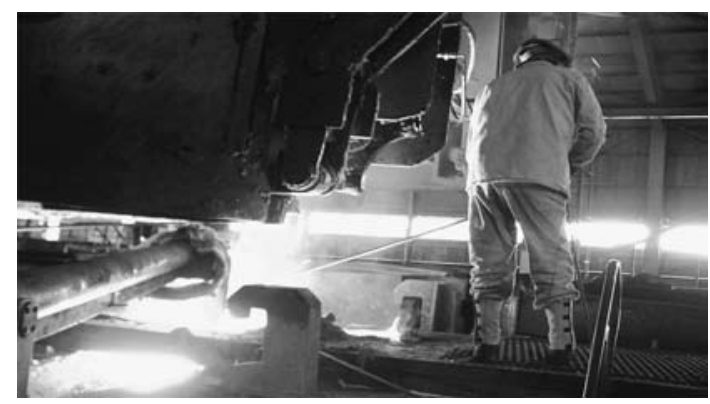

Figura 4. Medição da temperatura do aço líquido no distribuidor com lança de medição manual. 


\section{ANÁLISE GRÁFICA}

Na Figura 5 é mostrada a temperatura do aço líquido no distribuidor durante a operação.

Nesse gráfico visualiza-se a continuidade de medição da temperatura no aço líquido na troca da panela. Observa-se uma queda suave na temperatura durante a troca da panela abaixo de

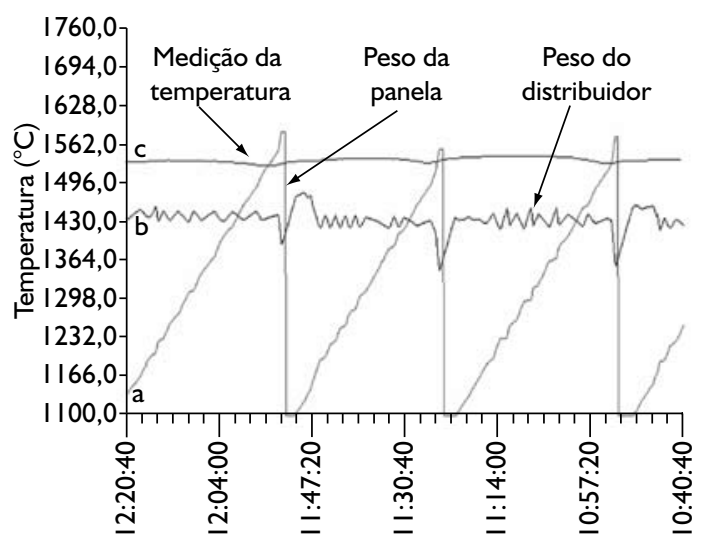

$\begin{array}{ll}\text { a) } 00111 \text { Peso panela } & \text { b) } 00101 \text { Peso distribuidor }\end{array}$ 5.7252 tons 25.650 tons

c) 00104 Temp. continua $1532,8^{\circ} \mathrm{C}$

Figura 5. Gráfico da medição contínua da temperatura do aço líquido.
I 8 toneladas. A ocorrência desse fato é devida ao baixo nível de aço no distribuidor, ficando a cerâmica total ou parcialmente fora do banho. Ao restabelecer o nível normal de operação no distribuidor ( 24 toneladas), a medição volta a se estabilizar.

Os momentos de troca de panelas estão representados nos picos da linha "Peso da Panela" no qual seu valor é máximo e o peso no distribuidor é mínimo.

O ganho está na continuidade da medição de temperatura durante essa troca que pode ser visualizada na constância da linha "Medição da Temperatura". O tempo necessário para atingir esta estabilidade está em torno de 5 minutos.

\section{CONCLUSÃO}

Dentre todas as tecnologias de medição analisadas, a utilização do pirômetro de fibra óptica foi a que apresentou o menor custo operacional e o maior grau de confiabilidade.

Esse desenvolvimento, na Usiminas, foi pioneiro e utilizou uma tecnologia de medição de temperatura disponível no mercado.

Adicionalmente ao uso do dispositivo com a tecnologia aplicada, é importante para uma medição confiável, desenvolver um projeto adequado ao ambiente de trabalho, minimizar as interferências geradas pelo processo, ajustar a medição com um padrão e escolher adequadamente o pirômetro óptico.

Enfim, trata-se de uma alternativa viável e atrativa levandose em consideração os ganhos operacionais, segurança e a relação custo/benefício do projeto.

\section{REFERÊNCIAS}

1 ECIL. Catálogo de pirometria, cabos de compensação e sensores de temperatura. São Paulo, 2000.

2 VESUVIUS. Manual do usuário do Sistema Accumetrix. Rio de Janeiro, 1994. p. 2-9.

3 AMERICAN SOCIETY FOR TESTING AND MATERIALS STANDARDS. ASTM E639: Standard test method for measuring total radiance temperature of heated surfaces using a radiation pyrometer. [S.I.], 2002.

4 RAYTEK. Marathon series FRI operator's manual. São Paulo, 2000.

5 LAND. Infrared application bulletin. [S.I.], 1999. p. I-2.

6 AMERICAN SOCIETY FOR TESTING AND MATERIALS STANDARDS. ASTM EI 256: Standard test methods for radiation thermometers. [S.I.], 1995.

Recebido em: 02/01/07

Aceito em: 29/05/07

Proveniente de: SEMINÁRIO DE AUTOMAÇÃO DE PROCESSOS, I0., 2006, Belo Horizonte. São Paulo: ABM, 2006. 